\title{
Video Article \\ 3D Hydrogel Scaffolds for Articular Chondrocyte Culture and Cartilage Generation
}

\author{
Piera Smeriglio* ${ }^{* 1}$, Janice H. Lai ${ }^{* 1,2}$, Fan Yang ${ }^{1,3}$, Nidhi Bhutani ${ }^{1}$ \\ ${ }^{1}$ Orthopaedic Surgery Department, Stanford University \\ ${ }^{2}$ Mechanical Engineering Department, Stanford University \\ ${ }^{3}$ Bioengineering Department, Stanford University \\ *These authors contributed equally
}

Correspondence to: Piera Smeriglio at pierasme@stanford.edu

URL: https://www.jove.com/video/53085

DOI: doi:10.3791/53085

Keywords: Bioengineering, Issue 104, Cartilage, hydrogels, tissue engineering, juvenile chondrocytes, adult chondrocytes, osteoarthritic chondrocytes

Date Published: 10/7/2015

Citation: Smeriglio, P., Lai, J.H., Yang, F., Bhutani, N. 3D Hydrogel Scaffolds for Articular Chondrocyte Culture and Cartilage Generation. J. Vis. Exp. (104), e53085, doi:10.3791/53085 (2015).

\section{Abstract}

Human articular cartilage is highly susceptible to damage and has limited self-repair and regeneration potential. Cell-based strategies to engineer cartilage tissue offer a promising solution to repair articular cartilage. To select the optimal cell source for tissue repair, it is important to develop an appropriate culture platform to systematically examine the biological and biomechanical differences in the tissue-engineered cartilage by different cell sources. Here we applied a three-dimensional (3D) biomimetic hydrogel culture platform to systematically examine cartilage regeneration potential of juvenile, adult, and osteoarthritic (OA) chondrocytes. The 3D biomimetic hydrogel consisted of synthetic component poly(ethylene glycol) and bioactive component chondroitin sulfate, which provides a physiologically relevant microenvironment for in vitro culture of chondrocytes. In addition, the scaffold may be potentially used for cell delivery for cartilage repair in vivo. Cartilage tissue engineered in the scaffold can be evaluated using quantitative gene expression, immunofluorescence staining, biochemical assays, and mechanical testing. Utilizing these outcomes, we were able to characterize the differential regenerative potential of chondrocytes of varying age, both at the gene expression level and in the biochemical and biomechanical properties of the engineered cartilage tissue. The 3D culture model could be applied to investigate the molecular and functional differences among chondrocytes and progenitor cells from different stages of normal or aberrant development.

\section{Video Link}

The video component of this article can be found at https://www.jove.com/video/53085/

\section{Introduction}

With its limited self-repair potential, human articular cartilage undergoes frequent irreversible damages. Extensive efforts are currently focused on the development of efficient cell-based approaches for treatment of articular cartilage injuries. The success of these cell-based therapies is highly dependent on the selection of an optimal cell source and the maintenance of its regenerative potential. Chondrocytes are a common cell source for cartilage repair, but they are limited in supply and can de-differentiate during in vitro expansion in 2D monolayer culture thereby limiting their generation of hyaline cartilage ${ }^{1}$.

The aim of this protocol is to establish a 3-dimensional hydrogel platform for an in vitro comparative study of human chondrocytes from different ages and disease state. Unlike conventional two-dimensional (2D) culture, three-dimensional (3D) hydrogels allow chondrocytes to maintain their morphology and phenotype and provides a physiologically relevant environment enabling chondrocytes to produce cartilage tissue ${ }^{2,3}$. In addition to providing a 3D physical structure for chondrocyte culture, hydrogels mimic the function of native cartilage extracellular matrix (ECM). Specifically, the inclusion of chondroitin sulfate methacrylate provides a potential reservoir for secreted paracrine factors ${ }^{4}$ and enables cellmediated degradation and matrix turnover ${ }^{5}$. Although many 3D hydrogel culture systems have been utilized widely in various studies including agarose and alginate gels, we have used a biomimetic 3D culture system that has some distinct advantages for chondrocyte culture. Chondroitin sulfate (CS) is an abundant component in articular cartilage and the PEG-CS hydrogels have been shown to maintain and even enhance chondrogenic phenotype and facilitate cell-mediated matrix degradation and turnover ${ }^{2,5}$. In addition, the mechanical properties of the hydrogel scaffold can be easily modulated by changing concentration of PEG and hence can be utilized to further enhance the regeneration potential of chondrocytes or a related cell type ${ }^{6,7}$. PEG/CSMA is also biocompatible and hence has the potential for a direct clinical application in cartilage defects for example. The limitation for this system is its complexity and the use of photopolymerization that can potentially affect cell viability as compared to simpler systems like agarose, however the advantages for the chondrocyte culture outweigh the potential limitations. 
The 3D hydrogel culture is compatible with conventional assay for evaluation of cell phenotype (gene expression, protein immunostaining) and functional outcome (quantification of cartilage matrix production, mechanical testing). This favorable 3D environment was tested to compare the tissue regeneration potential of human chondrocytes from three different aged populations in long-term 3D cultures.

The outcomes were evaluated via both phenotypic and functional assays. Juvenile, adult and OA chondrocytes showed differential responses in the 3D biomimetic hydrogel culture. After 3 and 6 weeks, chondrogenic gene expression was upregulated in juvenile and adult chondrocytes but was downregulated in OA chondrocytes. Deposition of cartilage tissue components including aggrecan, type II collagen, and glycosaminoglycan (GAG) was high for juvenile and adult chondrocytes but not for OA chondrocytes. The compressive moduli of the resulting cartilage constructs also exhibited similar trends. In conclusion, both juvenile and adult chondrocytes exhibited chondrogenic and cartilage matrix disposition up to 6 weeks of $3 \mathrm{D}$ culture in hydrogels. In contrast, osteoarthritic chondrocytes revealed a loss of cartilage phenotype and minimal ability to generate robust cartilage.

\section{Protocol}

All experiments were performed in accordance with the Stanford University Human subjects' guidelines and approved Institutional Review Board protocol.

\section{Articular Chondrocyte Isolation}

1. Obtain cartilage from tissue discarded during total knee arthroplasty and dissect as described previously ${ }^{8}$.

2. Visually select the medial or lateral femoral condyles of cartilage samples for smooth surfaces and make incision at the surface of the cartilage through a scalpel in order to remove $4-5 \mathrm{~mm}$ cartilage biopsies without affecting the subchondral bone.

3. Isolate chondrocytes from the extracellular matrix by enzymatic dissociation of the tissue with $\mathrm{O} / \mathrm{N}$ treatment with purified collagenase. Use 1:1 ratio of Collagenase II $(125 \mathrm{U} / \mathrm{ml})$ and Collagenase IV $(160 \mathrm{U} / \mathrm{ml})$ in chondrocyte growth media at $37^{\circ} \mathrm{C}$.

4. The following day, filter the digested tissue containing the dissociated cells through a $70 \mathrm{~mm}$ pore size Nylon mesh. Wash two times with 20 $\mathrm{ml}$ of DMEM/F12 and centrifuge at $600 \mathrm{xg}$ for $5 \mathrm{~min}$.

5. Plate the isolated chondrocytes at a density of $1 \times 10^{6}$ cells $/ 60 \mathrm{~mm}$ culture dish following re-suspension in DMEM/F12 supplemented with $10 \%$ Fetal Bovine Serum (FBS), $25 \mu \mathrm{g} / \mathrm{ml}$ ascorbate, $2 \mathrm{mM} \mathrm{L-glutamine,} \mathrm{antimicrobials} \mathrm{(100} \mathrm{U/ml} \mathrm{penicillin,} 100 \mu \mathrm{g} / \mathrm{ml} \mathrm{streptomycin,} \mathrm{and} 0.25$ $\mu \mathrm{g} / \mathrm{ml}$ fungizone). Keep the plates at $37^{\circ} \mathrm{C}$ and maintain the primary chondrocyte cultures as a high-density monolayer prior to encapsulation in biomimetic hydrogels.

\section{Biomimetic Hydrogel Fabrication}

NOTE: This method allows the synthesis of a biomimetic hydrogel containing poly(ethylene glycol diacrylate) (PEGDA, MW 5,000 g/mole), chondroitin sulfate-methacrylate (CS-MA) in DPBS. The addition of photoinitiator enables photoactivated crosslinking. The final hydrogel composition contains $7 \%$ weight/volume (w/v) of PEGDA, $3 \% \mathrm{w} / \mathrm{v}$ of CS-MA, and $0.05 \% \mathrm{w} / \mathrm{v}$ of photoinitiator.

1. Synthesize CS-MA by functionalizing CS polymer with methacrylate groups.

1. Prepare buffered solution of $50 \mathrm{mM}$ 2-morpholinoethanesulfonic acid (MES) and $0.5 \mathrm{M}$ sodium chloride ( $\mathrm{NaCl}$ ) by dissolving $1.952 \mathrm{~g}$ of MES and $5.84 \mathrm{~g}$ of NaCl in $200 \mathrm{ml}$ of deionized water $\left(\mathrm{DiH}_{2} \mathrm{O}\right)$. Dissolve $5 \mathrm{~g}$ of chondroitin sulfate sodium salt in the buffered solution.

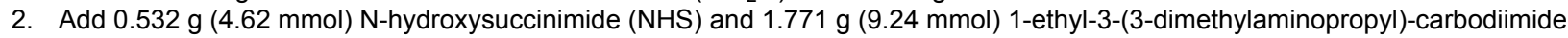
hydrochloride $(E D C)$ (molar ratio of NHS:EDC $=1: 2$ ) to the solution and stir for $5 \mathrm{~min}$.

3. Add $0.765 \mathrm{~g}(4.62 \mathrm{mmol})$ 2-aminoethyl methacrylate (AEMA) (molar ratio of NHS:EDC:AEMA $=1: 2: 1)$ to the solution and maintain the reaction at $\mathrm{RT}$ for $24 \mathrm{hr}$.

4. Purify the mixture by dialysis against deionized water $\left(\mathrm{diH}_{2} \mathrm{O}\right)$ for 4 days using dialysis tubing (12-14 kDa MWCO).

5. Filter the purified solution through a $0.22 \mu \mathrm{m}$ filter and place the open tubes containing the solution in the desiccator and vacuum $\mathrm{O} / \mathrm{N}$. Ensure that all tubes are protected from light. Store the dissolved polymer at $-20^{\circ} \mathrm{C}$ protected from light and moisture.

\section{Cell Encapsulation}

1. Autoclave the PCR film and cylindrical rods (for punching out the cell-laden $3 D$ hydrogels) and sterilize the custom-made cylindrical gel mold by submersion in $0.2 \mu \mathrm{m}$ filtered $70 \%$ ethanol in tissue culture hood under UV light.

2. Seal the bottom of the gel mold with autoclaved PCR film without air bubbles or gaps to prevent leaks. Keep it in a sterile $150 \mathrm{~mm}$ plate.

3. The day prior to cell encapsulation in biomimetic hydrogel, dissociate the high-density chondrocyte monolayer with $\mathrm{O} / \mathrm{N}$ treatment in Collagenase II and Collagenase IV solution. Use $125 \mathrm{U} / \mathrm{ml}$ for Collagenase II and $160 \mathrm{U} / \mathrm{ml}$ for Collagenase VI each in chondrocyte growth media at $37^{\circ} \mathrm{C}$.

4. Take a $50 \mathrm{ml}$ tube and add $5 \%$ weight/volume (w/v) poly(ethylene glycol diacrylate) (PEGDA, MW=5,000 g/mole), $3 \%$ w/v chondroitin sulfatemethacrylate (CS-MA), and $0.05 \% \mathrm{w} / \mathrm{v}$ photoinitiator in DPBS. Vortex the tube to mix the solution and keep it aside. Protect the tube from light.

5. Collect the dissociated cells in a $50 \mathrm{ml}$ Falcon tube, count and centrifuge at $460 \mathrm{xg}$ for $5 \mathrm{~min}$. Aspirate all the media from the cells tube and add the above mixed gel material to re-suspend cells at a density of $15 \times 10^{6}$ cells $/ \mathrm{ml}$. Mix it 30 times thoroughly while avoiding air bubbles formation.

6. Pipette $72 \mu \mathrm{l}$ of the cell-hydrogel suspension or hydrogel alone into the custom-made cylindrical gel mold and Induce gelation through exposure to UV light ( $365 \mathrm{~nm}$ wavelength) at $3 \mathrm{~mW} / \mathrm{m}^{2}$ for $5 \mathrm{~min}$. Prepare some hydrogel solution with no cell content. Use these constructs as negative controls for the future biochemical and mechanical testing. Measure the intensity of the UV light with a UV intensity meter. To adjust intensity, change the distance between the UV light source and the hydrogel scaffold during gelation. 
NOTE: Since the hydrogel contains CS, the acellular contribution is subtracted from the biochemical GAG content of the cell-laden hydrogels to represent only the cellular GAG.

7. Use a scalpel to cut the film for easy peel off and carefully remove it. With the help of the cylindrical rods, push out the gel into a 6 well plate with $5 \mathrm{ml}$ of sterile DPBS to wash off unpolymerized gel and loose cells.

8. Culture the cell-laden hydrogels in 24 well plates containing $1.5 \mathrm{ml}$ of chondrocyte growth media into each well. Use a disposable spatula to transfer the washed hydrogels into the well.

9. Assess cell viability by live dead staining $24 \mathrm{hr}$ post-encapsulation using a viability/cytotoxity kit. Culture the cell-laden hydrogels and the empty hydrogels for 3-6 weeks changing to fresh chondrocyte growth media every 2 days before harvesting for analyses.

\section{RNA Extraction and Gene Expression Analyses}

1. Carefully aspirate the media and put sterile PBS $1 \mathrm{X}$ on the cell-laden as well as the empty control hydrogels.

2. With the help of a sterile spatula, transfer each hydrogel to a clean $1.5 \mathrm{ml}$ eppendorf tube and add $250 \mu \mathrm{l}$ of Tri-reagent to each tube. Follow manufacturer's instructions to isolate RNA.

3. After RNA isolation and quantification use $1 \mu \mathrm{g}$ of it from each sample and perform a reversed transcription into cDNA using the High Capacity cDNA Reverse Transcription Kit.

4. For quantitative PCR use TaqMan Gene Expression Arrays for examination of the expression of your genes of interest. Normalize the gene expression levels internally to GAPDH.

5. For the PCR conditions include a 2 min incubation at $50^{\circ} \mathrm{C}$ to inactivate previous amplicons with uracil-DNA glycosylase, followed by a 10 min incubation at $95^{\circ} \mathrm{C}$ to activate the Taq polymerase. Then perform forty cycles of PCR, consisting of 15 sec at $95^{\circ} \mathrm{C}$, and 1 min at 60 ${ }^{\circ} \mathrm{C}$

\section{Biochemical Analyses}

1. For each cell-hydrogel constructs quantify DNA and sulfated glycosaminoglycan (sGAG) production as follow.

2. Carefully aspirate the media and put sterile PBS $1 \mathrm{X}$ on the cell-laden as well as the empty control hydrogels. Weigh an empty tube and put the hydrogel inside after blotting it on tissue paper and record the weight or wet weight of the hydrogels.

3. Freeze each hydrogel at $-20^{\circ} \mathrm{C}$ in a separate $1.5 \mathrm{ml}$ eppendorf tube for enzymatic digestion and later use an aliquot of the digested product to run Picogreen assay (for DNA) and Dimethyl-Methylene blue assay (for GAG).

4. Prepare the papainase solution for enzymatic digestion of the hydrogels.

1. First prepare the PBE buffer by weighing out $7.1 \mathrm{~g}$ of Sodium phosphate dibasic $\left(\mathrm{Na}_{2} \mathrm{HPO}_{4}\right)$ and $1.6 \mathrm{~g}$ of Ethylenediaminetetraacetic acid disodium salt $\left(E D T A-\mathrm{Na}_{2}\right)$. Dissolve in $500 \mathrm{ml}$ of $\mathrm{dH}_{2} \mathrm{O}$, adjust the $\mathrm{pH}$ to 6.5 and filter the purified solution through a $0.22 \mathrm{~mm}$ filter.

2. Dissolve $0.035 \mathrm{~g}$ of L-cysteine in $20 \mathrm{ml}$ of PBE buffer. Filter the solution and $100 \mu \mathrm{l}$ of sterile papain enzyme.

5. When ready to perform the assays, take the tubes from $-20^{\circ} \mathrm{C}$ and add $300 \mu$ of the ready papain solution to the frozen hydrogels. Crush the gel with a mortar and pestle and mix it well with the motor pestle.

6. Bring the volume to $500 \mu \mathrm{l}$ with additional papain solution. Perform the same procedure for the control gels. Incubate at $60{ }^{\circ} \mathrm{C}$ for $16 \mathrm{hr}{ }^{3,9}$ The solution containing the digested hydrogel can therefore used for DNA and GAG quantification. Measure the DNA content using the Picogreen assay with Lambda phage DNA as standard following manufacturer's protocol.

7. Quantify the sulfated-GAG content using the 1,9-dimethylmethylene blue (DMMB) dye-binding assay with shark chondroitin sulfate as standard ${ }^{10}$. Determine the GAG content by dividing the amount of GAG for the corresponding wet weight.

\section{Mechanical Testing}

1. Perform unconfined compression tests using an Instron 5944 testing system fitted with a $10 \mathrm{~N}$ load cell.

1. After the desired days of in vitro culture, perform the compression test on the cell-hydrogel scaffold and the acellular control hydrogels.

2. Submerge the specimens in a PBS bath at RT and compress at a rate of $1 \%$ strain/sec to a maximum strain of $15 \%{ }^{11,12}$ since the physiological strain experienced by cartilage tissue under loading condition has been reported to be $10-20 \%{ }^{13,14}$.

2. Create stress vs. strain curves and curve fit using a third order polynomial equation. Determine the compressive tangent modulus from the curve fit equation at strain values of $15 \%$.

\section{Representative Results}

Bioactive hydrogels containing PEG and CS moieties (Figure 1) represent an ideal platform for culture and maturation of human articular chondrocytes $^{2,3,5,7}$. Chondrocytes from different ages and disease states can be cultured with the described method and analyzed for their phenotype, gene expression and biochemical and mechanical properties of the cartilage tissue generated. Three chondrocyte samples, juvenile6 months, adult- 34 years and osteoarthritic- 74 years, were harvested after 3 weeks of culture in 3D biomimetic hydrogels. Gene expression analyses of normal chondrocytes, both juvenile and adult, showed an increase in the expression of the chondrocyte genes Col2a1 and Col6a1. On the contrary, diseased chondrocytes showed a dramatic decrease in Col2a1 while maintaining the expression of Col6a1 (Figure 2) showing a loss of chondrogenic phenotype despite being cultured in a favorable biomimetic environment.

The CS-PEG hydrogels also serve as a dynamic environment for the chondrocytes to proliferate, digest the pre-existing matrix of PEG and CS and deposit their pericellular and extracellular matrix, the main components of the mature cartilage tissue. Given these abilities, chondrocyte expansion after 3 weeks of in vitro culture can be estimated by quantification of DNA with the Picogreen dye. Comparative analysis of the three groups of cells show that the cell density of juvenile and adult populations was unchanged while OA chondrocytes exhibited a dramatic decrease compared to day 1 of culture. A loss of DNA content was also observed for OA but not other chondrocytes suggesting possible cell death during the long-term culture (Figure 3). The secreted matrix was quantified as sulfated-GAG content by DMMB dye-binding assay at the end point 
stage after 3 weeks of cultures. GAG content is normalized by the wet weight of the hydrogel as recorded before the enzymatic digestion and the acellular contribution is subtracted. As shown in Figure 4, chondrocytes deposited a similar significant amount of GAG during 3 weeks of culture in 3D hydrogels.

The presence of a physical scaffold allows the evaluation of biomechanical properties of the samples through unconfined compression tests

${ }^{2,3}$. While acellular hydrogels undergo a decrease in the compressive moduli, cell-laden constructs maintained the compressive moduli after 3 weeks in culture (Figure 4). The phenotypic, biochemical and biomechanical analyses described here are therefore useful for evaluating and understanding the potential of different chondrocyte populations for engineering cartilage.

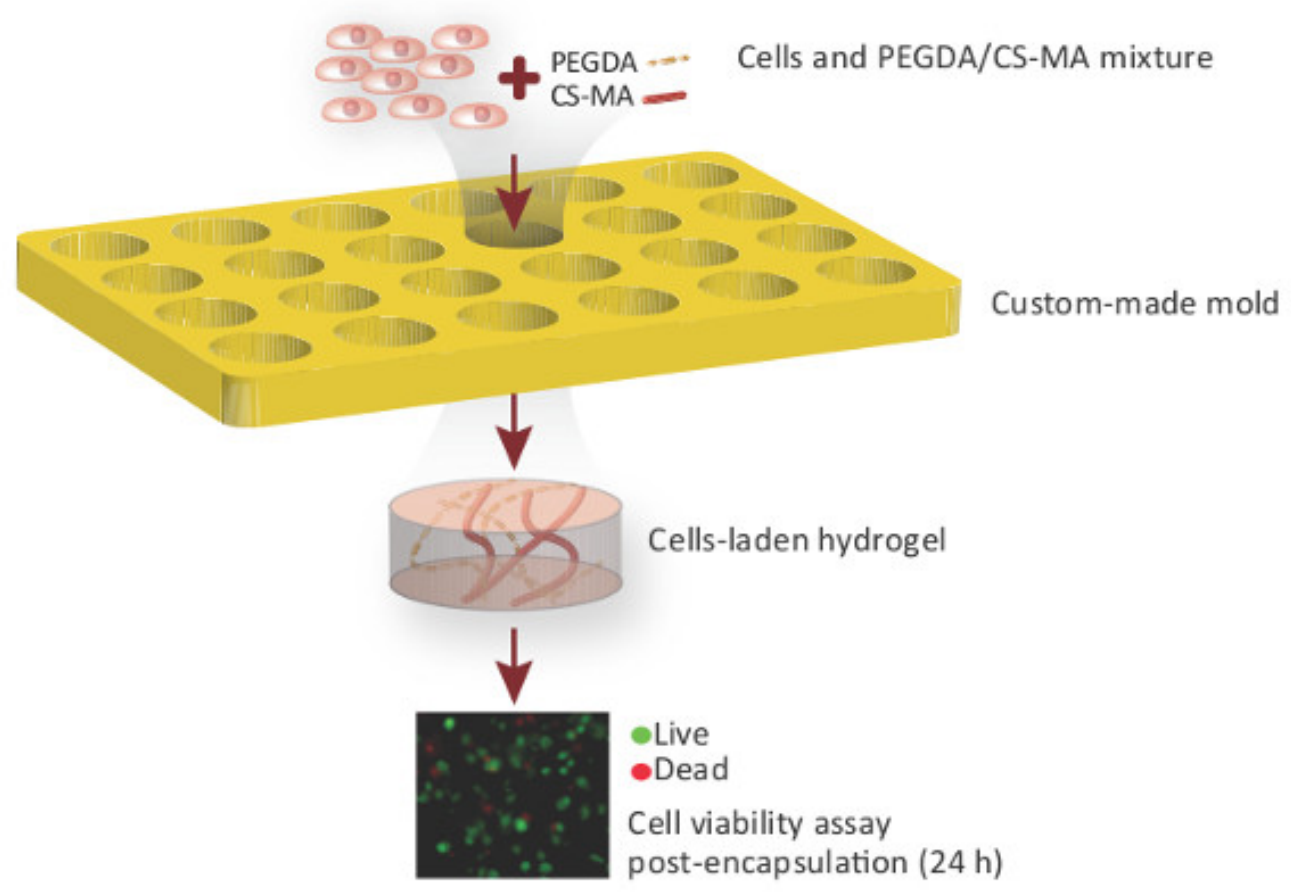

Figure 1. PEG-CS biomimetic hydrogels for 3D chondrocyte culture. Chondrocytes are resuspended in a mixture containing Poly(ethylene glycol diacrylate) (PEGDA) and chondroitin sulfate-methacrylate (CS-MA) and casted into the custom-made cylindrical gel mold. After UV exposure, solidified gels are collected from the molds and cell viability is assessed by live dead staining 24 hr post-encapsulation. Please click here to view a larger version of this figure.
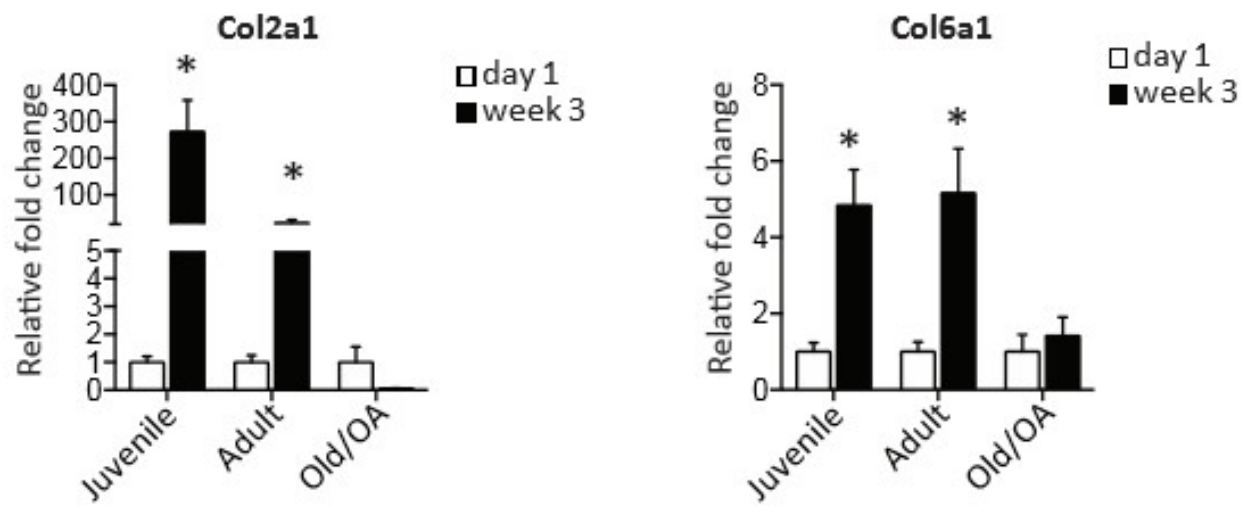

Figure 2. Expression of chondrogenic genes in human chondrocytes cultured in 3D biomimetic hydrogels. Quantitative gene expression of cartilage markers Col2a1 and Col6a1 by juvenile, adult and OA chondrocytes after 3 weeks of culture in 3D biomimetic hydrogels. Values are normalized to gene expression level at day 1 . Error bars represent mean \pm SD. $p^{*}<0.05$ as determined by a two-tailed Student $t$ test. Modified from Smeriglio et al. ${ }^{2}$ Please click here to view a larger version of this figure. 
Juvenile

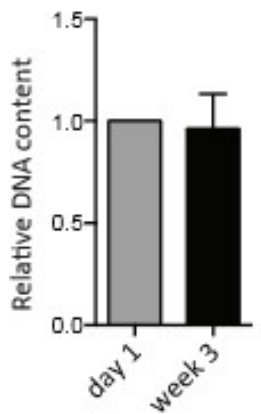

Adult

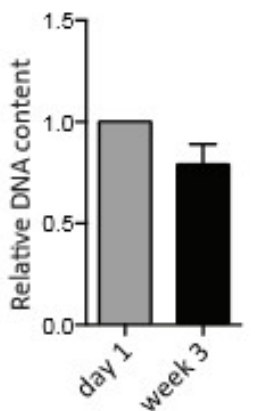

OA

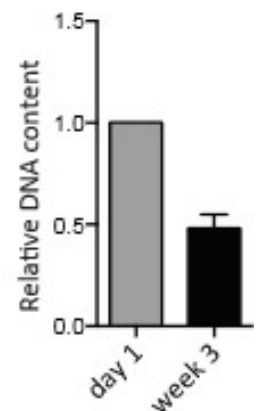

Figure 3. DNA content in human chondrocytes cultured in 3D biomimetic hydrogels. DNA quantification by Picogreen assay in juvenile, adult and OA chondrocytes after 3 weeks of culture in 3D biomimetic hydrogels. Values are normalized to DNA level at day 1. Please click here to view a larger version of this figure.

A

\section{Chondrocytes}

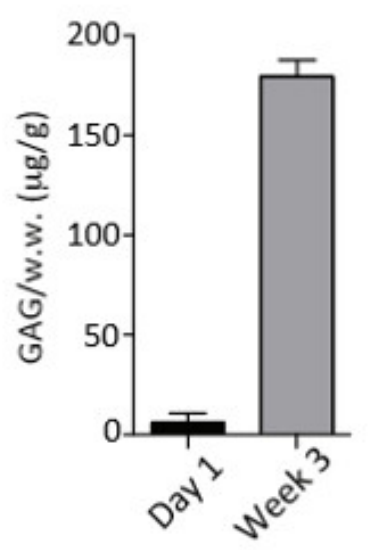

B

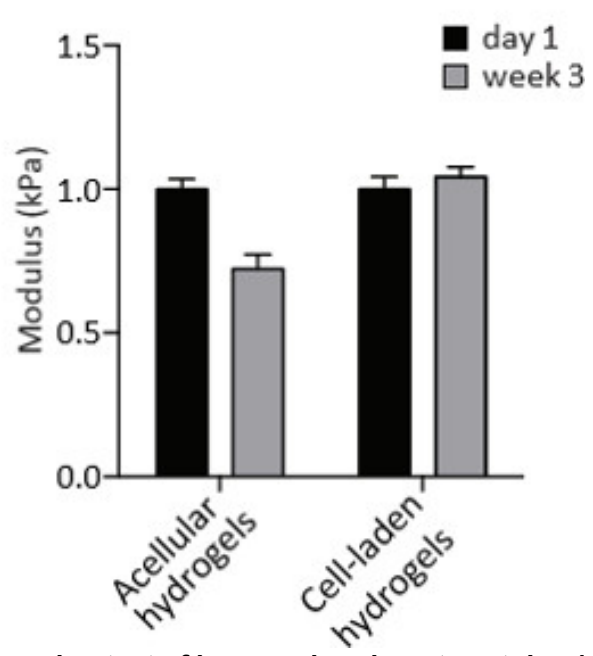

Figure 4. GAG content measurement and unconfined compression test of human chondrocytes at day 1 and after 3 weeks of culture in 3D biomimetic hydrogels. (A) GAG quantification by DMMB assay in chondrocytes at day 1 and after 3 weeks of culture in 3D biomimetic hydrogels. Values are normalized to wet weight (w.w.) and expressed as $\mu \mathrm{g} / \mathrm{g}$. (B) Compressive modulus (kPa) of acellular and cell-laden hydrogels at day 1 and after 3 weeks of culture in 3D biomimetic hydrogels. Please click here to view a larger version of this figure.

\section{Discussion}

As reported in this protocol, 3D hydrogels are able to maintain chondrocyte phenotype in culture, avoiding the process of cell dedifferentiation into fibrocartilage cells usually encountered with monolayer cultures ${ }^{15}$. Moreover, long-term cultures of the chondrocytes- hydrogel construct revealed a favorable environment that maintains the intrinsic cell features associated with age and disease.

The use of a 3D biomimetic hydrogel has several advantages. First, the inclusion of chondroitin sulfate (CS), a major component found in articular cartilage, enable cells to degrade the hydrogel matrix by secreting chondroitinase and lay down newly synthesized cartilage extracellular matrix $^{5,16}$. In addition, CS has been shown to have anti-inflammatory properties in the arthritic joint. The biomimetic hydrogel may also be used as a scaffolding material for cell delivery in cartilage repair, and may be chemically modified to facilitate better tissue-biomaterial integration $^{17,18}$.

The use of the PEG-CS hydrogels allows long-term cultures of chondrocytes and the evaluation of biochemical and mechanical properties. Here we show how this platform can be useful for the comparative analyses of various sources of differentiated chondrocytes in order to define the optimal cell type for cartilage engineering. Interestingly, chondrocytes encapsulated in hydrogels remain viable and proliferate according to their intrinsic abilities. The hydrogel composition supports, in fact, the growth of healthy juvenile and adult chondrocytes as shown in Figure 2. The composition and structure of the described hydrogels also promotes cartilage tissue formation as indicated by the deposition of a functional extracellular matrix assessed by glycosaminoglycan (GAG) quantification.

An additional advantage is that the chondrocyte-hydrogel constructs can be evaluated for the mechanical properties of the newly formed cartilage tissue. Note that the unconfined compression test should be performed on the acellular hydrogel for comparison. The hydrogels, in fact, have an intrinsic stiffness due to the rigidity of the CS moieties. Unconfined compression strain of $5-20 \%$ (at a strain rate $1 \% / \mathrm{s}$ ) can be applied for the mechanical testing of cartilage tissue ${ }^{11,12}$ since the physiological strain experienced by cartilage tissue under loading condition has been reported to be $10-20 \%{ }^{13,14}$. The response of both cell-laden and acellular hydrogels to mechanical testing was evaluated at the culture end-point. In the described example above we observed a comparable stiffness of the constructs containing adult and juvenile chondrocytes in 
contrast to the lower stiffness of the constructs containing OA chondrocytes. Such mechanical properties of the cell-hydrogel construct allow the assessment of the functional properties of the formed tissue giving an in-depth analysis of the cell maturation ability.

In conclusion, the use of the 3D biomimetic hydrogels to study the potential of different chondrocyte population to generate cartilage tissue can be widely applied. Besides the in vitro studies described here, in vivo transplantation of the cell-laden constructs can be envisioned to study cell maturation and regenerative potential in the physiological context. Further modifications of the hydrogel platform with additional biomimetic factors can also be envisioned to optimize chondrocyte proliferation and maturation.

\section{Disclosures}

The authors have nothing to disclose.

\section{Acknowledgments}

The authors would like to acknowledge Stanford Department of Orthopaedic Surgery and Stanford Coulter Translational Seed Grant for funding.

J.H.L. would like to thank National Science Foundation Graduate Fellowship and DARE Doctoral Fellowship for support.

\section{References}

1. Roberts, S., Menage, J., Sandell, L. J., Evans, E. H., Richardson, J. B. Immunohistochemical study of collagen types I and II and procollagen IIA in human cartilage repair tissue following autologous chondrocyte implantation. Knee. 16, 398-404 (2009).

2. Smeriglio, P., et al. Comparative Potential of Juvenile and Adult Human Articular Chondrocytes for Cartilage Tissue Formation in ThreeDimensional Biomimetic Hydrogels. Tissue engineering Part A. (2014).

3. Lai, J. H., Kajiyama, G., Smith, R. L., Maloney, W., Yang, F. Stem cells catalyze cartilage formation by neonatal articular chondrocytes in 3D biomimetic hydrogels. Scientific reports. 3, 3553 (2013).

4. Taipale, J., Keski-Oja, J. Growth factors in the extracellular matrix. FASEB journal : official publication of the Federation of American Societies for Experimental Biology. 11, 51-59 (1997).

5. Varghese, S., et al. Chondroitin sulfate based niches for chondrogenic differentiation of mesenchymal stem cells. Matrix Biol. 27, 12-21 (2008).

6. Park, J. S., et al. The effect of matrix stiffness on the differentiation of mesenchymal stem cells in response to TGF-beta. Biomaterials. 32 , 3921-3930 (2011).

7. Wang, T., Lai, J. H., Han, L. H., Tong, X., Yang, F. Chondrogenic Differentiation of Adipose-Derived Stromal Cells in Combinatorial Hydrogels Containing Cartilage Matrix Proteins with Decoupled Mechanical Stiffness. Tissue engineering Part A, doi:10.1089/ten.tea.2013.0531. Tissue engineering. Part A. (2014).

8. Smith, R. L., et al. Effects of intermittent hydrostatic pressure and BMP-2 on osteoarthritic human chondrocyte metabolism in vitro. J Orthop Res. 29, 361-368 (2011).

9. Buschmann, M. D., Gluzband, Y. A., Grodzinsky, A. J., Kimura, J. H., Hunziker, E. B. Chondrocytes in agarose culture synthesize a mechanically functional extracellular matrix. J Orthop Res. 10, 745-758 (1992).

10. Farndale, R. W., Buttle, D. J., Barrett, A. J. Improved quantitation and discrimination of sulphated glycosaminoglycans by use of dimethylmethylene blue. Biochim Biophys Acta. 883, 173-177 (1986).

11. Lai, J. H., Levenston, M. E. Meniscus and cartilage exhibit distinct intra-tissue strain distributions under unconfined compression. Osteoarthritis and cartilage / OARS, Osteoarthritis Research Society. 18, 1291-1299 (2010).

12. Li, L. P., Buschmann, M. D., Shirazi-Adl, A. Strain-rate dependent stiffness of articular cartilage in unconfined compression. Journal of biomechanical engineering. 125, 161-168 (2003).

13. Armstrong, C. G., Bahrani, A. S., Gardner, D. L. In vitro measurement of articular cartilage deformations in the intact human hip joint under load. The Journal of bone and joint surgery. American. 61, 744-755 (1979).

14. Macirowski, T., Tepic, S., Mann, R. W. Cartilage stresses in the human hip joint. Journal of biomechanical engineering. 116, 10-18 (1994).

15. Benya, P. D., Shaffer, J. D. Dedifferentiated chondrocytes reexpress the differentiated collagen phenotype when cultured in agarose gels. Cell. 30, 215-224 (1982).

16. Buckwalter, J. A., Mankin, H. J. Articular cartilage: tissue design and chondrocyte-matrix interactions.Instructional course lectures. 47, 477-486 (1998).

17. Wang, D. A., et al. Multifunctional chondroitin sulphate for cartilage tissue-biomaterial integration. Nat Mater.6, 385-392 (2007).

18. Simson, J. A., Strehin, I. A., Allen, B. W., Elisseeff, J. H. Bonding and fusion of meniscus fibrocartilage using a novel chondroitin sulfate bone marrow tissue adhesive. Tissue engineering. (Part A). 19, 1843-1851 (2013). 